\title{
Performance Research on Magnetotactic Bacteria Optimization Algorithm with the Best Individual-Guided Differential Interaction Energy
}

\author{
Hongwei Mo, Lili Liu, Jiao Zhao \\ Automation College, Harbin Engineering University, Harbin, China \\ Email: honwei2004@126.com, liulilikaoyan@163.com, zhaojiaoyouxiang@163.com
}

Received March 2015

\begin{abstract}
Magnetotactic bacteria optimization algorithm (MBOA) is a new optimization algorithm inspired by the characteristics of magnetotactic bacteria, which is a kind of polyphyletic group of prokaryotes with the characteristics of magnetotaxis that make them orient and swim along geomagnetic field lines. The original Magnetotactic Bacteria Optimization Algorithm (MBOA) and several new variants of MBOA mimics the interaction energy between magnetosomes chains to obtain moments for solving problems. In this paper, Magnetotactic Bacteria Optimization Algorithm with the Best Individual-guided Differential Interaction Energy (MBOA-BIDE) is proposed. We improved interaction energy calculation by using the best individual-guided differential interaction energy formation. We focus on analyzing the performance of different parameters settings. The experiment results show that the proposed algorithm is sensitive to parameters settings on some functions.
\end{abstract}

\section{Keywords}

Magnetotactic Bacteria, Nature Inspired Computing, Differential Interaction Energy, Parameters Settings

\section{Introduction}

The research of algorithms have been conducted many years, the field of algorithm is very mature now. Evolutionary algorithm (EA) is a very popular research field. The common evolutionary algorithms are genetic algorithm (GA), Differential Evolution (DE) [1], Particle Swarm Optimization (PSO) [2] and Bacterial Foraging Optimization algorithm (BFOA) [3] and so on.

Magnetotactic Bacteria Optimization Algorithm (MBOA) [4] [5] which is introduced by Mo is one of the modern heuristic algorithms and inspired by the magnetotactic bacteria. In nature, magnetotactic bacteria (MTBs) is a special kind of bacteria which have many micro magnetic particles named magnetosome in their bodies. 
These magnetic particles can generate moments to guide the bacteria to swim along geomagnetic field lines of the earth [6] [7]. In recent years, several improved MBOA, such as BMMBOA [8], MBOA-BR [9], MBOA-BT [10], PSMBA [11], MBMMA [12], have been proposed to modify the performance of MBOA. In the Moments of the Best Individual-based Magnetotactic Bacteria Optimization Algorithm (BMMBOA), similar to DE/best/1, the problem solutions are generated by moments mechanisms based on interaction energy among solutions [8]. In the Magnetotactic Bacteria Optimization Algorithm Based On Best-R and Scheme (MBOA-BR), similar to $\mathrm{DE} / \mathrm{best} / \mathrm{r}$ and scheme, it regulates the moments based on the information exchange between best individual's moments and some randomly one [9]. In the Magnetotactic Bacteria Optimization Algorithm based on Best-Target (MBOA-BT), similar to DE/best/target scheme, some cells will receive MTS information from the interaction between the local best one and the target one to balance the local search and global search [10]. In the Power Spectrum-Based Magnetotactic Bacteria Algorithm (PSMBA), it is based on the models of power spectra of the magnetic field noise produced by Brownian rotation of nonmotile bacteria in zero magnetic field [11]. In the Magnetotactic Bacteria Moment Migration Algorithm (MBMMA), the moments of relative good solutions can migrate each other to enhance the diversity of the MBMMA [12].

In this paper, we proposed a Magnetotactic Bacteria Optimization Algorithm with the Best Individual-guided Differential Interaction Energy (MBOA-BIDE) in order to overcome the shortcomings of complicated interaction energy calculation of the original MBOA and several new variants of MBOA and focus on the study of the effect of different parameters settings.

\section{Magnetotactic Bacteria Optimization Algorithm with the Best Individual-Guided Differential Interaction Energy (MBOA-BIDE)}

In the following, we briefly describe the basic operators and the main steps of MBOA-BIDE. MBOA-BIDE mainly has three steps and three main operators including moment generation, moment regulation, moment replacement.

\subsection{Interaction Distance}

First, in the algorithm, each solution is looked as a cell containing a magnetosome chain. At first we define $X_{\text {best }}$ stands for the best cell of the population in the current generation $t$. The distance of the cell $X_{i}$ and the best cell $X_{\text {best }}, D_{i}^{t}=\left(d_{i 1}^{t}, d_{i 2}^{t}, \ldots, d_{i n}^{t}\right)$, is calculated as follows: .

$$
D_{i}^{t}=X_{\text {best }}^{t}-X_{i}^{t}
$$

After that, we can get a distance matrix $D^{t}=\left(D_{1}^{t}, D_{2}^{t}, \ldots D_{i}^{t}, \ldots, D_{N}^{t}\right)^{\prime}=\left[\begin{array}{cccc}d_{11}^{t} & d_{12}^{t} & \cdots & d_{1 n}^{t} \\ d_{21}^{t} & d_{22}^{t} & \cdots & d_{2 n}^{t} \\ \vdots & \vdots & \cdots & \vdots \\ d_{N 1}^{t} & d_{N 2}^{t} & \cdots & d_{N n}^{t}\end{array}\right] . \quad N \quad$ is the size of cell population, $n$ stands for dimension of every cell.

\subsection{Moments Generation}

Based on the distances among cells, the interaction energy $E_{i}^{t}=\left(e_{i 1}^{t}, e_{i 2}^{t}, \ldots, e_{i j}^{t}, \ldots, e_{i n}^{t}\right)$ is defined as

$$
e_{i j}^{t}=c_{1} * d_{i j}^{t}+c_{2} * d_{p q}^{t}
$$

where the settings of $c_{1}$ and $c_{2}$ will be discussed in the next section. $d_{p q}^{t}$ stands for randomly selected variables from $D^{t} . p \in[1, N], q \in[1, n]$.

After obtaining interaction energy, the moments $M_{i}^{t}$ are generated as follows:

$$
M_{i}^{t}=\frac{E_{i}^{t}}{B}
$$

where the settings of $B$ will be discussed in the next section.

Suppose $M_{i}^{t}=\left(m_{i 1}^{t}, m_{i 2}^{t}, \ldots, m_{i j}^{t}, \ldots, m_{i n}^{t}\right)$, we can obtain a moment vector matrix $M^{t}=\left(M_{1}^{t}, M_{2}^{t}, \ldots M_{i}^{t}, \ldots, M_{N}^{t}\right)^{\prime}$ 


$$
=\left[\begin{array}{cccc}
m_{11}^{t} & m_{12}^{t} & \cdots & m_{1 n}^{t} \\
m_{21}^{t} & m_{22}^{t} & \cdots & m_{2 n}^{t} \\
\vdots & \vdots & \cdots & \vdots \\
m_{N 1}^{t} & m_{N 2}^{t} & \cdots & m_{N n}^{t}
\end{array}\right] .
$$

Then the total moments of a cell is regulated as follows:

$$
v_{i j}^{t}=x_{i j}^{t}+m_{l s}^{t} \times \text { rand }
$$

where $m_{l s}^{t}$ stands for the moment of a randomly selected MTS from $M^{t} . \quad l \in[1, N], s \in[1, n]$.

\subsection{Moments Regulation}

After moments generation, the moments regulation is realized as follows:

If rand $>0.5$, the moments in the cell are regulated as follows:

$$
u_{i j}^{t}=v_{\text {cbestj }}^{t}+\left(v_{\text {cbestj }}^{t}-v_{i j}^{t}\right) \times \text { rand }
$$

Otherwise, they are regulated as follows:

$$
u_{i j}^{t}=v_{i j}^{t}+\left(v_{c b e s t j}^{t}-v_{i j}^{t}\right) \times \text { rand }
$$

where $v_{\text {cbestj }}^{t}$ stands for the $j$ th dimension of current best cell $V_{\text {cbest }}^{t}$ in the current generation.

\subsection{Moments Replacement}

After the moments regulation, we set a replacement probability 0.5, some cells with worse fitness are replaced as follows:

if rand $>0.5$,

$$
x_{i j}^{t+1}=m_{l^{j}}^{t} \times \text { rand }
$$

where $l^{\prime}$ is a random number between 1 and $N . m_{l^{\prime} j}^{t}$ stands for the moment of a randomly selected MTS from $M_{l^{\prime}}^{t}$

\section{Parameters Settings}

To evaluate the performance of MBOA-BIDE, the experiments are carried out on 10 benchmark functions. In this section, the benchmark functions are presented firstly. Secondly, the simulation results obtained from different parameter settings are analyzed and discussed.

In all experiments, during each run, a maximum fitness evaluation of 200000 generations is used. To reduce statistical errors, each test is repeated 30 times independently and the mean results are used in the comparisons.

\subsection{Benchmark functions}

The ten basic benchmark problems summarised in Table 1, can be classified into two groups. The first five functions $f_{1}-f_{5}$ are unimodal functions. The unimodal functions here are used to test if MBOA-BIDE can maintain the fast-converging feature compared with the other methods. The next five functions $f_{6}-f_{10}$ are multimodal functions with many local optima. These functions can be used to test the global search ability of the algorithm in avoiding premature convergence.

\subsection{The effect of population size $N$}

We set MBOA-BIDE with different population size ( $N=10,40,50,100,150$ and 200). The results of different population size are presented in Table 2. From Table 2, we can see that population size $N$ with 40 is providing the best results in eight of the ten selected functions.

In this study, MBOA-BIDE with different population size $N$ are ranked based on their mean performances. They are ranked according to their performances using a standard competition ranking scheme. In competition ranking, algorithms receive the same rank if their performances are same. The next performing algorithm is 
Table 1. Benchmark functions.

\begin{tabular}{|c|c|c|c|}
\hline Function & Range & $\mathrm{D}$ & Formulation \\
\hline$f_{1}:$ Sphere & {$[-100,100]$} & 30 & $f(x)=\sum_{i=1}^{n} x_{i}^{2}$ \\
\hline$f_{2}:$ Schwefel 2.22 & {$[-10,10]$} & 30 & $f(x)=\sum_{i=1}^{n}\left|x_{i}\right|+\prod_{i=1}^{n}\left|x_{i}\right|$ \\
\hline$f_{3}:$ Schwefel 1.2 & {$[-100,100]$} & 30 & $f(x)=\sum_{i=1}^{n}\left(\sum_{j=1}^{i} x_{j}\right)^{2}$ \\
\hline$f_{4}:$ Quartic Noise & {$[-1.28,1.28]$} & 30 & $f(x)=\sum_{i=1}^{n} i x_{i}^{4}+\operatorname{random}[0,1)$ \\
\hline$f_{5}:$ Rosenbrock & {$[-30,30]$} & 30 & $f(x)=\sum_{i=1}^{n-1}\left[100\left(x_{i+1}-x_{i}^{2}\right)^{2}+\left(x_{i}-1\right)^{2}\right]$ \\
\hline$f_{6}:$ Rastrigin & {$[-5.12,5,12]$} & 30 & $f(x)=\sum_{i=1}^{n}\left[x_{i}^{2}-10 \cos \left(2 \pi x_{i}\right)+10\right.$ \\
\hline$f_{7} \quad$ Generalized Schwefel & {$[-500,500]$} & 30 & $f(x)=\sum_{i=1}^{n}-x_{i} \sin \left(\sqrt{\left|x_{i}\right|}\right.$ \\
\hline$f_{8}$ : Foxholes & {$[-65.536,65.536]$} & 2 & $f(x)=\left[\frac{1}{500}+\sum_{j=1}^{25} \frac{1}{j+\sum_{i=1}^{2}\left(x_{i}-a_{i j}\right) 6}\right]^{-1}$ \\
\hline$f_{9}$ : Sixhump & {$[-5,5]$} & 2 & $f(x)=4 x_{1}^{2}-2.1 x_{1}^{4}+\frac{1}{3} x_{1}^{6}+x_{1} x_{2}-4 x_{2}^{2}+4 x_{2}^{4}$ \\
\hline$f_{10}:$ Goldstein price & {$[-2,2]$} & 2 & $\begin{array}{l}f(x)=\left[\begin{array}{c}1+\left(x_{1}+x_{2}+1\right)^{2} \\
\left(19-14 x_{1}+3 x_{1}^{2}-14 x_{2}+6 x_{1} x_{2}+3 x_{2}^{2}\right)\end{array}\right] \\
{\left[\begin{array}{c}30+\left(2 x_{1}-3 x_{2}\right)^{2} \\
\left.18-32 x_{1}+12 x_{1}^{2}+48 x_{2}-36 x_{1} x_{2}+27 x_{2}^{2}\right)\end{array}\right]}\end{array}$ \\
\hline
\end{tabular}

assigned a rank with a gap (gap is determined based on the number of equally performing algorithms). Table $\mathbf{3}$ provides the ranks of the different population size and the average rank for all the functions based on mean performances. Based on the average ranking, the order of performance obtained is $N=40$ followed by $N=50$, $N=10, N=100, N=150$ and $N=200$ respectively.

Figure 1 presents the histograms that indicate the number of times each population size $N$ have achieved the ranks in the range of 1 to 6 . It can be seen that $N=40$ achieves the top rank as compared to the other different population size.

\subsection{The effect of magnetic field $B$}

To study the effects of $B$ in MBOA-BIDE, we use $f_{5}, f_{7}$ and $f_{8}$ for testing the performance of MBOABIDE. Firstly we suppose $B$ is constant $(B=1,3,5,7,10)$, and study the effect of $B$ on test functions. Secondly, we also study the effect of $B$ varying with generation increases as follows:

- $B$ is linearly increases from 1 to10 ( $B=1$ - 10 LINER).

- $B$ is exponentially increases from 1 to 10 ( $B=1-10$ EXP )

- $B$ is linearly increases from 1 to 100 ( $B=1$ - 100 LINER)

- $B$ is exponentially increases from 1 to 100 ( $B=1-100$ EXP).

The results are shown in Table 4. From Table 4, for $f_{5}$, we can see that when $B$ is constant, $B=10$, the method can achieve better performance, when $B=1$ - 100 LINER can achieve better performance on $f_{5}$. For $f_{7}$, when $B=3$ and $B=1-100 \mathrm{EXP}$, the method can achieve better performance. For $f_{8}$, we can see that when $B=1$ and $B=1$ - 10 LINER can achieve better performance.

Figure 2 Presents the line chart and histograms that indicate the mean, best and median values each $B$ have achieved for $f_{7}$, respectively. From Figure 2, we can see when $B=1-100 \mathrm{EXP}$, MBOA-BIDE achieve the 


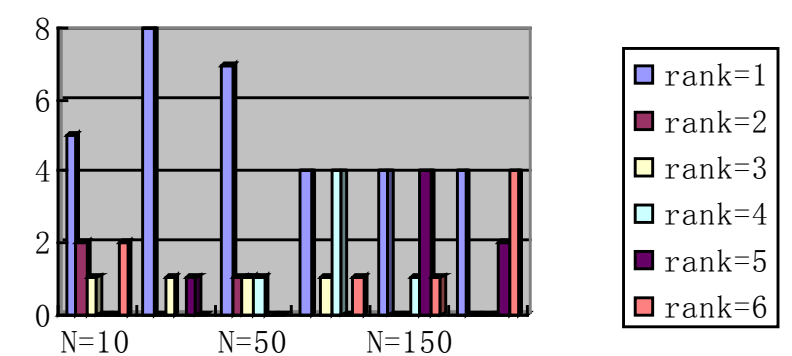

Figure 1. Histogram of individual mean ranks.

Table 2. Statistical results obtained by MBOA-BIDE with different population size $N$.

\begin{tabular}{|c|c|c|c|c|c|c|c|}
\hline Func. & & $N=10$ & $N=40$ & $N=50$ & $N=100$ & $N=150$ & $N=200$ \\
\hline \multirow{3}{*}{$f_{1}$} & Mean & 0 & 0 & 0 & 0 & $2.5201 \mathrm{e}^{-}-254$ & $4.5896 \mathrm{e}-181$ \\
\hline & Dev & 0 & 0 & 0 & 0 & 0 & 0 \\
\hline & Rank & 1 & 1 & 1 & 1 & 5 & 6 \\
\hline \multirow{3}{*}{$f_{2}$} & Mean & 0 & 0 & 0 & $4.3137 \mathrm{e}-209$ & $3.3968 \mathrm{e}-129$ & $5.5820 \mathrm{e}-91$ \\
\hline & Dev & 0 & 0 & 0 & 0 & $6.3405 e^{-129}$ & $1.2236 \mathrm{e}-90$ \\
\hline & Rank & 1 & 1 & 1 & 4 & 5 & 6 \\
\hline \multirow{3}{*}{$f_{3}$} & Mean & 0 & 0 & 0 & $3.0876 \mathrm{e}-312$ & $5.5975 \mathrm{e}^{-182}$ & $1.0773 \mathrm{e}^{-124}$ \\
\hline & Dev & 0 & 0 & 0 & 0 & 0 & $3.8100 \mathrm{e}^{-}-124$ \\
\hline & Rank & 1 & 1 & 1 & 4 & 5 & 6 \\
\hline \multirow{3}{*}{$f_{4}$} & Mean & $1.4263 e^{-05}$ & $2.2258 \mathrm{e}-05$ & $1.1082 e-05$ & $2.7951 e^{-05}$ & $2.2837 \mathrm{e}-05$ & $2.7755 e^{-05}$ \\
\hline & Dev & $1.0928 \mathrm{e}-05$ & $1.7502 \mathrm{e}-05$ & $1.1717 \mathrm{e}-05$ & $2.8866 \mathrm{e}-05$ & $1.5794 \mathrm{e}-05$ & 3. $4894 \mathrm{e}-05$ \\
\hline & Rank & 2 & 3 & 1 & 6 & 4 & 5 \\
\hline \multirow{3}{*}{$f_{5}$} & Mean & 21.4943 & 21.3003 & 22.1453 & 23.6719 & 24.2513 & 24.6209 \\
\hline & Dev & 0.7394 & 0.4264 & 0.3933 & 0.2229 & 0.2143 & 0.1610 \\
\hline & Rank & 2 & 1 & 3 & 4 & 5 & 6 \\
\hline \multirow{3}{*}{$f_{6}$} & Mean & 0 & 0 & 0 & 0 & 0 & 0 \\
\hline & Dev & 0 & 0 & 0 & 0 & 0 & 0 \\
\hline & Rank & 1 & 1 & 1 & 1 & 1 & 1 \\
\hline \multirow{3}{*}{$f_{7}$} & Mean & $-1.1199 \mathrm{e}+04$ & $-1.1808 \mathrm{e}+04$ & $-1.1535 e+04$ & $-1.0843 e+04$ & $-1.0664 \mathrm{e}+04$ & $-1.0807 e+04$ \\
\hline & Dev & $1.0496 \mathrm{e}+03$ & $1.0526 \mathrm{e}+03$ & $1.5453 \mathrm{e}+03$ & $1.9108 \mathrm{e}+03$ & $1.7327 \mathrm{e}+03$ & $1.5578 \mathrm{e}+03$ \\
\hline & Rank & 3 & 1 & 2 & 4 & 6 & 5 \\
\hline \multirow{3}{*}{$f_{8}$} & Mean & 7.8914 & 2.5827 & 2.4521 & 1.1949 & 0.9980 & 0.9980 \\
\hline & Dev & 4.0483 & 3.3070 & 3.6346 & 1.0783 & $4.1884 \mathrm{e}^{-}-12$ & $5.8272 \mathrm{e}-12$ \\
\hline & Rank & 6 & 5 & 4 & 3 & 1 & 1 \\
\hline \multirow{3}{*}{$f_{9}$} & Mean & -1.03162842 & -1.03162845 & -1.03162845 & -1.03162845 & -1.03162845 & -1.03162845 \\
\hline & Dev & $3.2639 \mathrm{e}-08$ & $6.5144 \mathrm{e}-10$ & $9.0922 \mathrm{e}-10$ & $3.3173 \mathrm{e}-10$ & $4.8852 \mathrm{e}-10$ & $7.3936 \mathrm{e}-10$ \\
\hline & Rank & 6 & 1 & 1 & 1 & 1 & 1 \\
\hline \multirow{3}{*}{$f_{10}$} & Mean & 3.0000 & 3.00000 & 3.0000 & 3.00000 & 3.00000 & 3.00000 \\
\hline & Dev & $3.7073 e^{-07}$ & $5.7118 e^{-08}$ & 3.7689e-08 & $5.3505 e-08$ & $2.3598 \mathrm{e}-08$ & $3.1246 \mathrm{e}-08$ \\
\hline & Rank & 1 & 1 & 1 & 1 & 1 & 1 \\
\hline
\end{tabular}




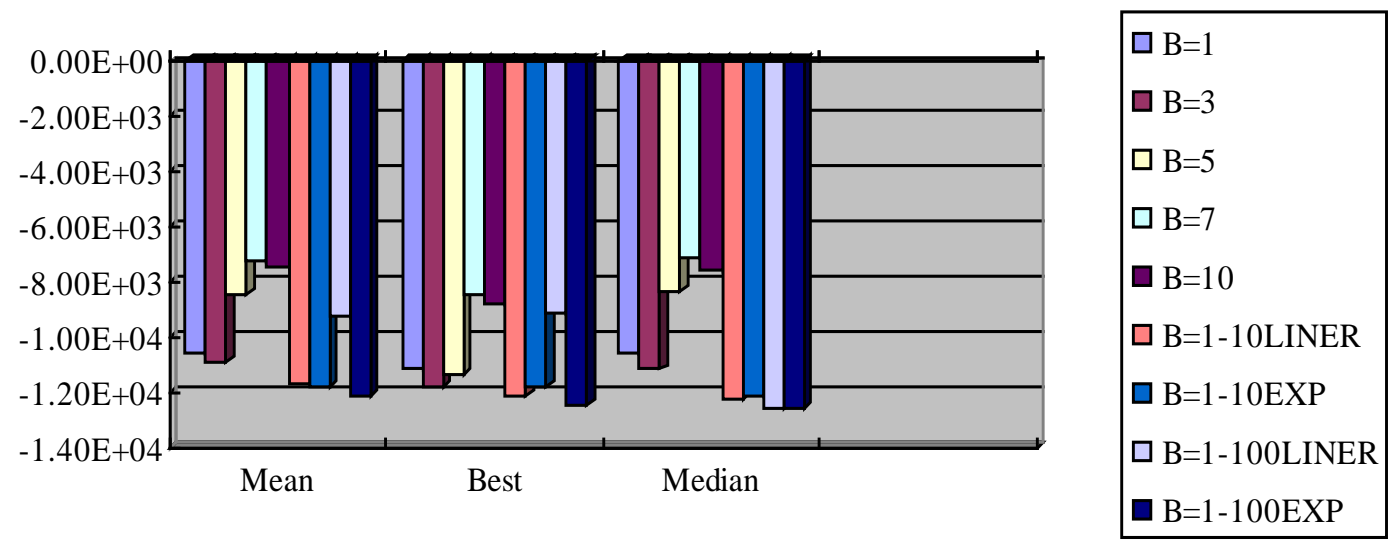

Figure 2. Histogram of statistical results of MBOA-BIDE with different $B$ values

Table 3. Rank table for the mean values.

\begin{tabular}{|c|c|c|c|c|c|c|}
\hline Fun & $\mathrm{N}=10$ & $N=40$ & $\mathrm{~N}=50$ & $\mathrm{~N}=100$ & $\mathrm{~N}=150$ & $N=200$ \\
\hline$f_{1}$ & 1 & 1 & 1 & 1 & 5 & 6 \\
\hline$f_{2}$ & 1 & 1 & 1 & 4 & 5 & 6 \\
\hline$f_{3}$ & 1 & 1 & 1 & 4 & 5 & 6 \\
\hline$f_{4}$ & 2 & 3 & 1 & 6 & 4 & 5 \\
\hline$f_{5}$ & 2 & 1 & 3 & 4 & 5 & 6 \\
\hline$f_{6}$ & 1 & 1 & 1 & 1 & 1 & 1 \\
\hline$f_{7}$ & 3 & 1 & 2 & 4 & 6 & 5 \\
\hline$f_{8}$ & 6 & 5 & 4 & 3 & 1 & 1 \\
\hline$f_{9}$ & 6 & 1 & 1 & 1 & 1 & 1 \\
\hline$f_{10}$ & 1 & 1 & 1 & 1 & 1 & 1 \\
\hline Avg. rank & 2.4 & 1.6 & 1.6 & 2.9 & 3.4 & 3.8 \\
\hline
\end{tabular}

Table 4. Statistical results obtained by MBOA-BIDE with different B values.

\begin{tabular}{|c|c|c|c|c|c|c|}
\hline Fun & & Mean & Median & Dev & Best & Worst \\
\hline \multirow{9}{*}{$f_{5}$} & $B=1$ & 26.9030 & 26.9095 & 0.1675 & 26.5374 & 27.2250 \\
\hline & $B=3$ & 21.6974 & 21.8007 & 0.4795 & 20.6532 & 22.7105 \\
\hline & $B=5$ & 21.2422 & 21.2866 & 0.4115 & 19.8061 & 21.8931 \\
\hline & $B=7$ & 21.1473 & 21.2003 & 0.4291 & 19.7336 & 22.0915 \\
\hline & $B=10$ & 20.9895 & 21.0589 & 0.3426 & 19.8434 & 21.5301 \\
\hline & $B=1-10$ LINER & 21.2495 & 21.3203 & 0.4827 & 20.4088 & 22.3778 \\
\hline & $B=1-10 \mathrm{EXP}$ & 20.3502 & 20.3270 & 0.9573 & 18.1462 & 21.9417 \\
\hline & $B=1-100$ LINER & 19.3093 & 19.3314 & 0.1863 & 18.7989 & 19.6055 \\
\hline & $\mathrm{B}=1-100 \mathrm{EXP}$ & 21.0213 & 21.0466 & 0.3493 & 20.1838 & 21.4598 \\
\hline
\end{tabular}




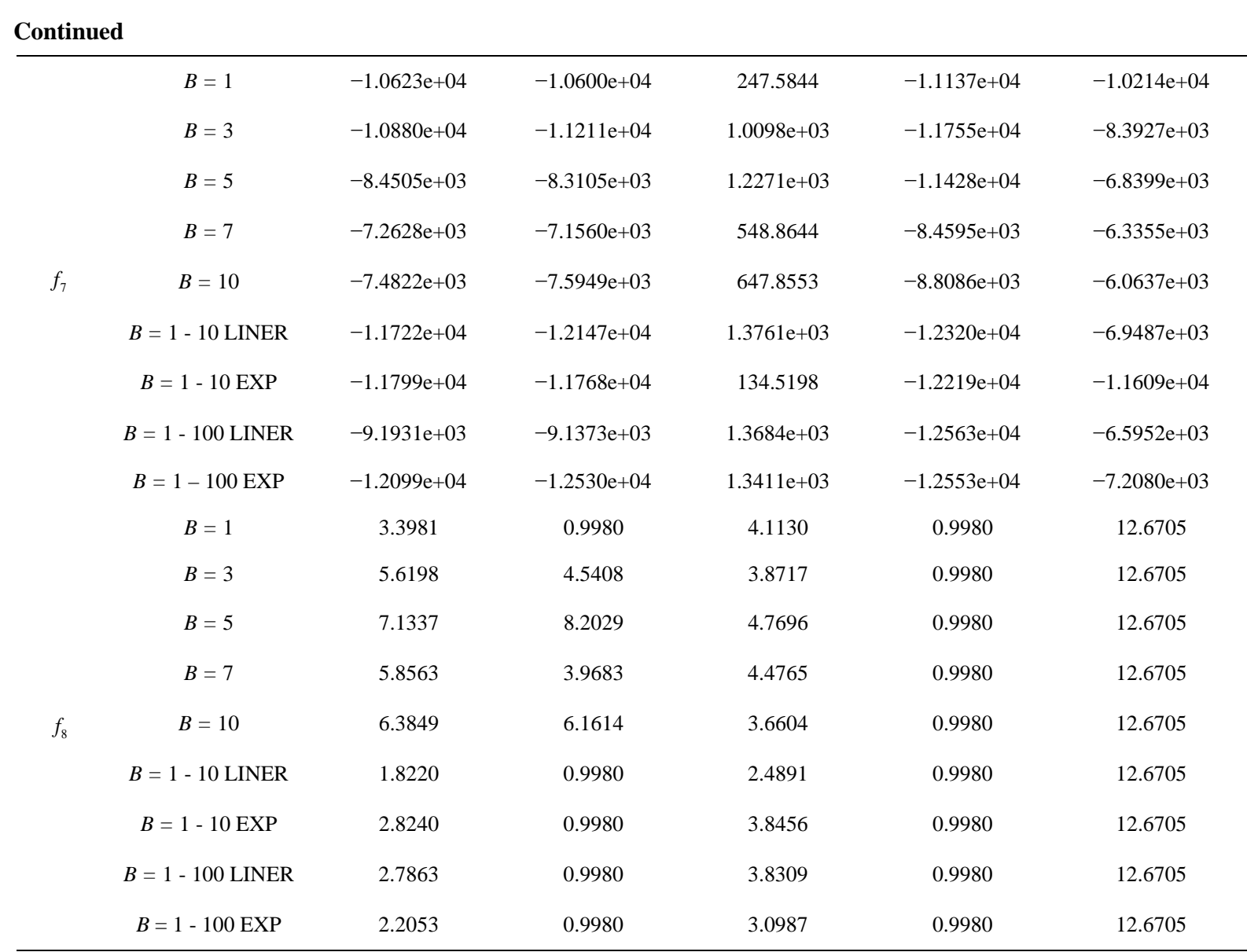

performance on $f_{7}$.

\subsection{The Effect of $c_{1}$ and $c_{2}$}

Firstly we suppose that $c_{1}$ and $c_{2}$ is constant, and study the effect of $c_{1}$ and $c_{2}$ on three test functions. We set $c_{1}+c_{2}=1$, and $c_{1}$ with different values $(0.1,0.3,0.5,0.7,0.9)$. Secondly, we also study the effect of $c_{1}$ and $c_{2}$ varying with generation increases. The parameter settings are as follows:

- $c_{1}=0-1 \mathrm{~L}: c_{1}$ is linearly increases from 0 to1, $c_{2}=1-c_{1}$;

- $c_{2}=0-1 \mathrm{~L}: c_{2}$ is linearly increases from0 to1. $c_{1}=1-c_{2}$;

- $c_{1}=0.1-1 \mathrm{E}: c_{1}$ is exponentially increases from 0.1 to $1, c_{2}=1-c_{1}$;

- $c_{2}=0.1-1 \mathrm{E}: c_{2}$ is exponentially increases from 0.1 to $1, c_{1}=1-c_{2}$;

- $c_{1}=0-2 \mathrm{~L}: c_{1}$ is linearly increases from 0 to2, $c_{2}=2-c_{1}$;

- $c_{2}=0-2 \mathrm{~L}: c_{2}$ is linearly increases from0 to2, $c_{1}=2-c_{2}$;

The statistical results are shown in Table 5.

From Table 5 and Table 6, we can see that for $f_{5}, c_{2}=0.1-1 \mathrm{E}$ obtain the best performance. For $f_{7}$ and $f_{8}, c_{1}=0-2 \mathrm{~L}$ obtain the best performance. and based on the average ranking, the order of performance obtained is $c_{2}=0-1 \mathrm{~L}$ followed by $c_{1}=0-2 \mathrm{~L}, c_{1}=0-1 \mathrm{~L}, c_{2}=0-2 \mathrm{~L}, c_{1}=0.3, c_{1}=0.1, c_{1}=0.1-$ $1 \mathrm{E}, c_{1}=0.9, c_{1}=0.7, c_{2}=0.1-1 \mathrm{E}$ and $c_{1}=0.5$, respectively.

\section{Conclusion}

In this paper, by analyzing the performance of different parameters settings of MBOA-BIDE, we can see that when $N=40-50, B$ is exponentially increases from 1 to $100, c_{2}$ is linearly increases from 0 to $1 . c_{1}=1$ - $c_{2}$, MBOA-BIDE obtain the better performance. The experiment results show that the proposed algorithm is 
Table 5. Statistical results obtained by MBOA-BIDE with different $C_{1}$ and $C_{2}$ values.

\begin{tabular}{|c|c|c|c|c|c|c|c|}
\hline Fun & & Mean & Median & Dev & Best & Worst & Rank \\
\hline \multirow{11}{*}{$f_{5}$} & $c_{1}=0.1$ & 21. 3901 & 21.4491 & 0.2710 & 20.6426 & 21.8700 & 9 \\
\hline & $c_{1}=0.3$ & 21.0181 & 21.0926 & 0.3837 & 20.1813 & 21.6728 & 6 \\
\hline & $c_{1}=0.5$ & 21.0303 & 21.0385 & 0.3369 & 20.2972 & 21.7053 & 7 \\
\hline & $c_{1}=0.7$ & 20.9564 & 20.9955 & 0.3636 & 20.0590 & 21.5185 & 5 \\
\hline & $c_{1}=0.9$ & 21.3102 & 21.3443 & 0.2975 & 20.4737 & 21.8988 & 8 \\
\hline & $c_{1}=0-1 \mathrm{~L}$ & 21.0213 & 21.0466 & 0.3493 & 20.1838 & 21.4598 & 3 \\
\hline & $c_{2}=0-1 \mathrm{~L}$ & 21.0500 & 21.0776 & 0.4023 & 20.2052 & 21.7740 & 4 \\
\hline & $c_{1}=0.1-1 \mathrm{E}$ & 20.8318 & 20.8450 & 0.3135 & 20.2436 & 21.6018 & 2 \\
\hline & $c_{2}=0.1-1 \mathrm{E}$ & 20.7453 & 20.7975 & 0.3485 & 19.8905 & 21.3564 & 1 \\
\hline & $c_{1}=0-2 \mathrm{~L}$ & 21.8727 & 22.0773 & 0.6619 & 19.7414 & 22.8133 & 11 \\
\hline & $c_{2}=0-2 \mathrm{~L}$ & 21.7704 & 21.9754 & 0.5780 & 20.3235 & 22.8893 & 10 \\
\hline \multirow{11}{*}{$f_{7}$} & $c_{1}=0.1$ & $-1.2352 \mathrm{e}+04$ & $-1.2532 \mathrm{e}+04$ & 985.4425 & $-1.2549 \mathrm{e}+04$ & $-7.1345 e+03$ & 4 \\
\hline & $c_{1}=0.3$ & $-1.2406 \mathrm{e}+04$ & $-1.2533 e+04$ & 690.3886 & $-1.2547 \mathrm{e}+04$ & $-8.7509 e+03$ & 3 \\
\hline & $c_{1}=0.5$ & $-1.2295 \mathrm{e}+04$ & $-1.2536 \mathrm{e}+04$ & 912.3674 & $-1.2548 \mathrm{e}+04$ & $-8.8768 \mathrm{e}+03$ & 6 \\
\hline & $c_{1}=0.7$ & $-1.2170 \mathrm{e}+04$ & $-1.2535 e+04$ & $1.1179 \mathrm{e}+03$ & $-1.2556 \mathrm{e}+04$ & $-8.6381 e+03$ & 7 \\
\hline & $c_{1}=0.9$ & $-1.2105 \mathrm{e}+04$ & $-1.2529 \mathrm{e}+04$ & $1.3193 e+03$ & $-1.2548 \mathrm{e}+04$ & $-7.3420 \mathrm{e}+03$ & 8 \\
\hline & $c_{1}=0-1 \mathrm{~L}$ & $-1.2099 \mathrm{e}+04$ & $-1.2530 \mathrm{e}+04$ & $1.3411 \mathrm{e}+03$ & $-1.2553 e+04$ & $-7.2080 \mathrm{e}+03$ & 9 \\
\hline & $c_{2}=0-1 \mathrm{~L}$ & $-1.2417 \mathrm{e}+04$ & $-1.2535 e+04$ & 646.0001 & $-1.2552 \mathrm{e}+04$ & $-8.9972 \mathrm{e}+03$ & 2 \\
\hline & $c_{1}=0.1-1 \mathrm{E}$ & $-1.2091 \mathrm{e}+04$ & $-1.2534 \mathrm{e}+04$ & $1.3939 \mathrm{e}+03$ & $-1.2548 \mathrm{e}+04$ & $-6.6607 e+03$ & 10 \\
\hline & $c_{2}=0.1-1 \mathrm{E}$ & $-1.1775 e+04$ & $-1.2533 e+04$ & $1.7649 \mathrm{e}+03$ & $-1.2547 \mathrm{e}+04$ & $-6.7527 e+03$ & 11 \\
\hline & $c_{1}=0-2 \mathrm{~L}$ & $-1.2430 \mathrm{e}+04$ & $-1.2436 e+04$ & 34.6550 & $-1.2488 \mathrm{e}+04$ & $-1.2349 \mathrm{e}+04$ & 1 \\
\hline & $c_{2}=0-2 \mathrm{~L}$ & $-1.2297 \mathrm{e}+04$ & $-1.2434 \mathrm{e}+04$ & 763.0817 & $-1.2498 \mathrm{e}+04$ & $-8.2595 \mathrm{e}+03$ & 5 \\
\hline \multirow{11}{*}{$f_{8}$} & $c_{1}=0.1$ & 2.2362 & 0.9980 & 3.3244 & 0.9980 & 12.6705 & 4 \\
\hline & $c_{1}=0.3$ & 2.7849 & 0.9980 & 3.7353 & 0.9980 & 12.6705 & 8 \\
\hline & $c_{1}=0.5$ & 4.2983 & 0.9980 & 4.6656 & 0.9980 & 12.6705 & 11 \\
\hline & $c_{1}=0.7$ & 2.9363 & 0.9980 & 3.7453 & 0.9980 & 12.6705 & 9 \\
\hline & $c_{1}=0.9$ & 2.4267 & 0.9980 & 3.7530 & 0.9980 & 12.6705 & 6 \\
\hline & $c_{1}=0-1 \mathrm{~L}$ & 2.2053 & 0.9980 & 3.0987 & 0.9980 & 12.6705 & 3 \\
\hline & $c_{2}=0-1 \mathrm{~L}$ & 2.3454 & 0.9980 & 3.5817 & 0.9980 & 12.6705 & 5 \\
\hline & $c_{1}=0.1-1 \mathrm{E}$ & 2.7436 & 0.9980 & 3.6848 & 0.9980 & 12.6705 & 7 \\
\hline & $c_{2}=0.1-1 \mathrm{E}$ & 3.1675 & 0.9980 & 4.0601 & 0.9980 & 12.6705 & 10 \\
\hline & $c_{1}=0-2 \mathrm{~L}$ & 1.0102 & 0.9980 & 0.0563 & 0.9980 & 1.2898 & 1 \\
\hline & $c_{2}=0-2 \mathrm{~L}$ & 1.5118 & 0.9980 & 2.0119 & 0.9980 & 12.6705 & 2 \\
\hline
\end{tabular}


Table 6. Rank table for the mean values.

\begin{tabular}{|c|c|c|c|c|}
\hline \multirow{2}{*}{$\mathrm{c}_{1}, \mathrm{C}_{2}$} & \multicolumn{3}{|c|}{ Individual ranking of benchmark functions } & \multirow[t]{2}{*}{ Avg. RANK (R) } \\
\hline & $f_{5}$ & $f_{7}$ & $f_{8}$ & \\
\hline$c_{1}=0.1$ & 9 & 4 & 4 & 5.67 \\
\hline$c_{1}=0.3$ & 6 & 3 & 8 & 5.67 \\
\hline$c_{1}=0.5$ & 7 & 6 & 11 & 8 \\
\hline$c_{1}=0.7$ & 5 & 7 & 9 & 7 \\
\hline$c_{1}=0.9$ & 8 & 8 & 6 & 6.67 \\
\hline$c_{1}=0-1 \mathrm{~L}$ & 3 & 9 & 3 & 5 \\
\hline$c_{2}=0-1 \mathrm{~L}$ & 4 & 2 & 5 & 3.67 \\
\hline$c_{1}=0.1-1 \mathrm{E}$ & 2 & 10 & 7 & 6.33 \\
\hline$c_{2}=0.1-1 \mathrm{E}$ & 1 & 11 & 10 & 7.33 \\
\hline$c_{1}=0-2 \mathrm{~L}$ & 11 & 1 & 1 & 4.33 \\
\hline$c_{2}=0-2 \mathrm{~L}$ & 10 & 5 & 2 & 5.67 \\
\hline
\end{tabular}

sensitive to parameters settings on some functions.

\section{Acknowledgements}

This work is partially supported by the National Natural Science Foundation of China under Grant No.61075113, the Excellent Youth Foundation of Heilongjiang Province of China under Grant No. JC201212, the Fundamental Research Funds for the Central Universities No. HEUCFX041306.

\section{References}

[1] Storn, R. and Price, K. (1997) Differential Evolution-A Simple and Efficient Heuristic for Global Optimization over Continuous Spaces. Journal of Global Optimization, 11, 341-359. http://dx.doi.org/10.1023/A:1008202821328

[2] Kennedy, J. and Eberhart, R. (1995) Particle Swarm Optimization. IEEE International Conference on Neural Networks, Piscataway, NJ, 1942-1948. http://dx.doi.org/10.1109/ICNN.1995.488968

[3] Müeller, S., Marchetto, J., Airaghi, S. and Koumoutsakos, P. (2002) Optimization Based on Bacterial Chemotaxis. IEEE Transactions on Evolutionary Computation, 6, 16-29. http://dx.doi.org/10.1109/4235.985689

[4] Mo, H.W. (2012) Research on Magnetotactic Bacteria Optimization Algorithm. The 5th International Conference on Advanced Computational Intelligence (ICACI 2012), Nanjing, 423-427. http://dx.doi.org/10.1109/ICACI.2012.6463198

[5] Mo, H.W. and Xu, L.F. (2013) Magnetotactic Bacteria Optimization Algorithm for Multimodal Optimization. IEEE Symposium on Swarm Intelligence (SIS), Singapore, 240-247.

[6] Faivre, D. and Schuler, D. (2008) Magnetotactic Bacteria and Magnetosomes. Chemical Reviews, 108, 4875-4898. http://dx.doi.org/10.1021/cr078258w

[7] Mitchell, J.G. and Kogure, K. (2006) Bacterial Motility: Links to the Environment and a Driving Force for Microbial Physics. FEMS Microbiol. Ecol., 55, 3-16. http://dx.doi.org/10.1111/j.1574-6941.2005.00003.X

[8] Mo, H.W., Liu, L.L., Xu, L.F. and Zhao, Y.Y. (2014) Performance Research on Magnetotactic Bacteria Optimization Algorithm Based on the Best Individual. The 6th International Conference on Bio-inspired Computing (BICTA 2014), Wuhan, 318-322.

[9] Mo, H.W. and Geng, M.J. (2014) Magnetotactic Bacteria Optimization Algorithm Based on Best-r and Scheme. 6th Naturei and Biologically Inspired Computing, Porto Portgal, 59-64.

[10] Mo, H.W. and Liu, L.L. (2014) Magnetotactic Bacteria Optimization Algorithm Based on Best-Target Scheme. International Conference on Nature Computing and Fuzzy Knowledge, Xiamen, 103-114. 
http://dx.doi.org/10.1109/ICNC.2014.6975877

[11] Mo, H.W., Liu, L.L. and Xu, L.F. (2014) A Power Spectrum Optimization Algorithm Inspired by Magnetotactic Bacteria. Neural Computing and Applications, 25, 1823-1844. http://dx.doi.org/10.1007/s00521-014-1672-3

[12] Mo, H.W., Liu, L.L. and Geng, M.J. (2014) A New Magnetotactic Bacteria Optimization Algorithm Based on Moment Migration. 2014 International Conference on Swarm Intelligence, Hefei, 103-114.

http://dx.doi.org/10.1007/978-3-319-11857-4_12 